\title{
Outcomes of acute coronary syndromes in coronavirus disease 2019
}

\author{
Victoria L. Cammann ${ }^{1} \cdot$ Konrad A. Szawan $^{1} \cdot$ Fabrizio D'Ascenzo ${ }^{2} \cdot$ Sebastiano Gili $^{3}$ - Sara Dreiding ${ }^{1}$. \\ Michael Würdinger ${ }^{1} \cdot$ Robert Manka $^{1} \cdot$ Barbara E. Stähli ${ }^{1}$ - Erik W. Holy ${ }^{1} \cdot$ Patrick Siegrist $^{1}$ - Philipp Jakob ${ }^{1}$. \\ Philippe Meyer ${ }^{4}$. Mario lannaccone ${ }^{5}$ - Emanuela Di Simone ${ }^{5}$. Gioel Gabrio Secco ${ }^{6}$. Matteo Saccocci ${ }^{7}$. Luca Bettari $^{8}$. \\ Alfonso lelasi ${ }^{9} \cdot$ Maurizio Tespili $^{9} \cdot$ Giorgio Quadri $^{10} \cdot$ Ferdinando Varbella $^{10} \cdot$ Sergio Raposeiras-Roubin ${ }^{11}$. \\ Emad Abu-Assi ${ }^{11}$. Massimo Mancone ${ }^{12} \cdot$ Gennaro Sardella $^{12} \cdot$ Fabio Infusino $^{12}$. Francesco Fedele ${ }^{12}$. \\ Giuseppe Patti ${ }^{13} \cdot$ Marco Mennuni $^{14} \cdot$ Andrea Rognoni $^{14} \cdot$ Mario Bollati $^{15} \cdot$ Luca Olivotti $^{16} \cdot$ Stefano Cordone $^{17}$. \\ Stefano Carugo ${ }^{18}$. Lucia Barbieri $^{18}$. Luca Gaido $^{19} \cdot$ Massimo Giammaria $^{19}$. Alfonso Gambino ${ }^{20}$. \\ Maurizio D'Amico ${ }^{20}$ - Alessandro Galluzzo ${ }^{5}$. Fabrizio $\mathrm{Ugo}^{5}$. Daniela Trabattoni ${ }^{3}$. Ovidio De Filippo ${ }^{2}$. \\ Gaetano Maria De Ferrari ${ }^{2}$. Carmine Vecchione ${ }^{21}$ - Rodolfo Citro ${ }^{21}$ • Jelena R. Ghadri ${ }^{1}$. Christian Templin ${ }^{1}$ (C)
}

Sirs:

Coronavirus disease 2019 (COVID-19), caused by the severe acute respiratory syndrome coronavirus 2 (SARS-CoV-2), is a fast spreading disease with high morbidity and mortality [1]. COVID-19 can contribute to severe myocardial injury,

Victoria L. Cammann, Konrad A. Szawan, Jelena R. Ghadri and Christian Templin contributed equally to this work.

Christian Templin

Christian.Templin@usz.ch

1 Andreas Grüntzig Heart Catheterization Laboratories and Acute Cardiac Care, University Heart Center, Department of Cardiology, University Hospital Zurich, Raemistrasse 100, 8091 Zurich, Switzerland

2 Division of Cardiology, Department of Medical Sciences, AOU Città Della Salute E Della Scienza, University of Turin, Turin, Italy

3 Centro Cardiologico Monzino, IRCCS, Milan, Italy

4 Service de Cardiologie, Hôpitaux Universitaires de Genève, Geneva, Switzerland

5 Department of Cardiology, San Giovanni Bosco Hospital, Turin, Italy

6 Interventional Cardiology and Cardiac Surgery Unit, Azienda Ospedaliera SS Antonio E Biagio E Cesare Arrigo, Alessandria, Italy

7 Cardiac Surgery Unit, Cardiovascular Department - Poliambulanza Foundation Hospital, Brescia, Italy

8 Interventional Cardiology Unit, Cardiovascular Department - Poliambulanza Foundation Hospital, Brescia, Italy

9 Clinical and Interventional Cardiology Unit, Sant'Ambrogio Cardio-Thoracic Center, Milan, Italy ultimately culminating in acute coronary syndromes (ACS) [2]. Clinical features and outcomes of patients with SARSCoV-2 associated ACS have not been elucidated, yet.

In a multicenter study, COVID-19 positive patients diagnosed with angiographically confirmed ACS between February 19 and April 92020 at 17 sites in Italy, Spain, and

10 Cardiology Unit, Rivoli Hospital, Turin, Italy

11 Cardiology Department, University Hospital Alvaro Cunqueiro, Vigo, Spain

12 Department of Clinical Internal, Anesthesiological and Cardiovascular Sciences, Sapienza University of Rome, Rome, Italy

13 University of Eastern Piedmont, Interventional Cardiology, Maggiore Della Carità Hospital of Novara, Novara, Italy

14 Interventional Cardiology, Maggiore Della Carità Hospital of Novara, Novara, Italy

15 Cardiology Division, Ospedale Maggiore, Lodi, Italy

16 Department of Cardiology, Ospedale Santa Corona, Pietra Ligure, Savona, Italy

17 Department of Cardiology, Ospedale San Paolo, Savona, Italy

18 Division of Cardiology, ASST Santi Paolo E Carlo, Milan, Italy

19 Division of Cardiology, Maria Vittoria Hospital, Turin, Italy

20 Interventional Cardiology, S. Croce Hospital of Moncalieri, Turin, Italy

21 Heart Department, University Hospital "San Giovanni Di Dio E Ruggi D’Aragona”, Salerno, Italy 
Table 1 Characteristics of ACS Patients

\begin{tabular}{|c|c|c|c|}
\hline & $\begin{array}{l}\text { COVID-19 positive } \\
N=45\end{array}$ & $\begin{array}{l}\text { COVID-19 negative } \\
N=76\end{array}$ & $P$ value \\
\hline \multicolumn{4}{|l|}{ Demographics } \\
\hline Male sex-no./total no. $(\%)$ & $37 / 45(82.2)$ & $59 / 76(77.6)$ & 0.55 \\
\hline Age (years) & $69.7 \pm 11.1(N=45)$ & $65.8 \pm 10.7(N=76)$ & 0.06 \\
\hline BMI $\left(\mathrm{kg} / \mathrm{m}^{2}\right)$ & $26.5 \pm 3.2(N=44)$ & $27.7 \pm 4.9(N=74)$ & 0.11 \\
\hline \multicolumn{4}{|l|}{ ACS type } \\
\hline STE-ACS & $27 / 45(60.0)$ & $43 / 76(56.6)$ & 0.71 \\
\hline NSTE-ACS & $18 / 45(40.0)$ & $33 / 76(43.4)$ & 0.71 \\
\hline \multicolumn{4}{|l|}{ Symptoms on admission—no./total no. (\%) } \\
\hline Chest pain & $34 / 45(75.6)$ & $54 / 71(76.1)$ & 0.95 \\
\hline Dyspnea & $23 / 45(51.1)$ & $14 / 71(19.7)$ & $<0.001$ \\
\hline \multicolumn{4}{|l|}{ Cardiac biomarkers-median (IQR) } \\
\hline Troponin maximum—factor increase in ULN" & $97.36(33.44-411.78) N=43$ & $139.46(17.16-410.14) N=76$ & 0.79 \\
\hline Creatine kinase maximum-factor increase in ULN & $6.55(1.39-20.96) N=27$ & $4.16(0.94-10.48) N=76$ & 0.14 \\
\hline BNP maximum—factor increase in $\mathrm{ULN}^{\$}$ & $2.56(0.92-22.26) N=20$ & $4.03(1.62-11.05) N=72$ & 0.80 \\
\hline \multicolumn{4}{|l|}{ Inflammatory markers-median (IQR) } \\
\hline CRP maximum (mg/l) & $15.20(7.95-60.68) N=36$ & $26.00(6.80-62.00) N=75$ & 0.59 \\
\hline WBC maximum $\left(10^{-} 3 / \mu \mathrm{l}\right)$ & $11.94(9.44-16.58) N=44$ & $11.16(8.42-15.56) N=75$ & 0.15 \\
\hline \multicolumn{4}{|l|}{ Vital signs-mean $\pm \mathrm{SD}$} \\
\hline Heart rate on admission (beats/min) & $82.4 \pm 16.0(N=37)$ & $79.5 \pm 15.7(N=76)$ & 0.37 \\
\hline Systolic blood pressure on admission $(\mathrm{mmHg})$ & $131.6 \pm 26.6(N=45)$ & $134.0 \pm 27.4(N=76)$ & 0.64 \\
\hline Diastolic blood pressure on admission $(\mathrm{mmHg})$ & $78.1 \pm 16.1(N=45)$ & $77.9 \pm 14.6(N=76)$ & 0.94 \\
\hline $\operatorname{LVEF}(\%)$ & $42.5 \pm 11.4(N=43)$ & $44.7 \pm 13.1(N=53)$ & 0.40 \\
\hline \multicolumn{4}{|l|}{ ECG on admission } \\
\hline Sinus rhythm-no./total no. (\%) & $41 / 45(91.1)$ & $70 / 74(94.6)$ & 0.48 \\
\hline QTc (ms) & $430.2 \pm 28.0(N=31)$ & $435.9 \pm 34.5(N=74)$ & 0.42 \\
\hline \multicolumn{4}{|l|}{ Cardiovascular risk factors/comorbidities-no./total no. (\%) } \\
\hline Arterial hypertension & $36 / 45(80.0)$ & $39 / 76(51.3)$ & 0.002 \\
\hline Diabetes mellitus & $12 / 44(27.3)$ & $19 / 76(25.0)$ & 0.78 \\
\hline Hypercholesterolemia & $19 / 45(42.2)$ & $42 / 76(55.3)$ & 0.17 \\
\hline Cancer & $2 / 45(4.4)$ & $6 / 76(7.9)$ & $0.71 *$ \\
\hline Cerebrovascular disease & $3 / 45(6.7)$ & $7 / 76(9.2)$ & $0.74 *$ \\
\hline COPD/asthma & $2 / 45(4.4)$ & $7 / 76(9.2)$ & $0.48^{*}$ \\
\hline Coronary artery disease & $13 / 45(28.9)$ & $22 / 76(28.9)$ & 1.0 \\
\hline Renal disease & $5 / 45(11.1)$ & $10 / 76(13.2)$ & 0.74 \\
\hline \multicolumn{4}{|l|}{ Medication on admission—no./total no. (\%) } \\
\hline ACE inhibitor & $15 / 45(33.3)$ & $15 / 71(21.1)$ & 0.14 \\
\hline AT antagonist & $7 / 45(15.6)$ & $20 / 71(28.2)$ & 0.12 \\
\hline Beta-blocker & $16 / 45(35.6)$ & $18 / 71(25.4)$ & 0.24 \\
\hline Calcium-channel antagonist & $8 / 45(17.8)$ & $13 / 71(18.3)$ & 0.94 \\
\hline Statin & $16 / 45(35.6)$ & 29/71 (40.8) & $0.70 *$ \\
\hline Coumarin & $1 / 45(2.2)$ & $4 / 71(5.6)$ & 0.64 \\
\hline Direct oral anticoagulant & $1 / 45(2.2)$ & $1 / 71(1.4)$ & $1.0^{*}$ \\
\hline \multicolumn{4}{|l|}{ COVID-19 specific therapy-no./total no. (\%) } \\
\hline Hydroxychloroquine & $24 / 45(53.3)$ & & \\
\hline Remdesivir & $1 / 45(2.2)$ & & \\
\hline Lopinavir/Ritonavir & $12 / 45(26.7)$ & & \\
\hline Baricitinib & $1 / 45(2.2)$ & & \\
\hline Tocilizumab & $3 / 45(6.7)$ & & \\
\hline
\end{tabular}


Table 1 (continued)

COVID-19 positive

$N=45$
COVID-19 negative

$N=76$

Acute cardiac care treatment-no./total no. (\%)

Catecholamine use

Invasive or non-invasive ventilation

Cardiopulmonary resuscitation

In-hospital death—no./total no. (\%)

$9 / 37(24.3)$
$17 / 45(37.8)$
$6 / 37(16.2)$
$12 / 44(27.3)^{\circ}$

$12 / 76(15.8)$

$12 / 76(15.8)$

$13 / 76(17.1)$

$6 / 76(7.9)$

$P$ value

$A C E$ angiotensin converting enzyme, $A C S$ acute coronary syndrome, $A T$ angiotensin, $B M I$ body mass index, $B N P$ brain natriuretic peptide, $C O P D$ chronic obstructive pulmonary disease, COVID-19 coronavirus disease 2019, CRP c-reactive protein, $E C G$ electrocardiogram, IQR interquartile range, $L V E F$ left ventricular ejection fraction, NSTE non-ST-segment elevation, $Q T C$ QT time corrected for heart rate, $S D$ standard deviation, STE ST-segment elevation, $U L N$ upper limit of the normal, $W B C$ white blood cell count

"Including upper limits of troponin $\mathrm{T}$, high-sensitivity troponin $\mathrm{T}$ and troponin $\mathrm{I}$

${ }^{\$}$ Including upper limits of brain natriuretic peptide and the $\mathrm{N}$-terminal of prohormone brain natriuretic peptide

${ }^{\circ}$ One patient was still hospitalized at time of performing statistical analysis

*Fisher's exact test

Switzerland were compared to COVID-19 negative ACS patients from the University Hospital Zurich. In addition, patients with ST-segment elevation (STE)-ACS COVID 19 positive vs. COVID-19 negative were compared as well as patients with non-ST-segment elevation (NSTE)-ACS COVID-19 positive vs. COVID-19 negative.

Out of 4702 patients with COVID-19, 45 (0.96\%) had ACS, of which 27 (60.0\%) had STE-ACS and 18 (40.0\%) NSTE-ACS. Single vessel disease was present in 25 patients (55.6\%) of COVID-19 positive ACS and multi vessel disease in 20 patients (44.4\%), respectively. All patients received percutaneous coronary intervention.

COVID-19 positive ACS patients were more likely to present with dyspnea $(51.1 \%$ vs. $19.7 \% ; P<0.001)$ and arterial hypertension $(80.0 \%$ vs. $51.3 \% ; P=0.002)$, while other patients' characteristics were largely comparable to COVID-19 negative ACS patients (Table 1). Of note, inhospital mortality was more than 3 times higher in COVID19 positive ACS patients than in COVID-19 negative ACS patients $(27.3 \%$ vs. $7.9 \% ; P=0.004$, Table 1$)$. Furthermore, when stratifying patients according to the presence or absence of ST-segment elevation, COVID-19 positive patients with STE-ACS had higher mortality rates compared to COVID-19 negative STE-ACS patients (33.3\% vs. 9.3\%; $P=0.024)$ and also COVID-19 positive patients with NSTEACS showed numerically higher mortality rates compared to COVID-19 negative NSTE-ACS patients $(17.6 \%$ vs. $6.1 \%$; $P=0.32$ ). Importantly, 9 out of 12 (75\%) deceased COVID19 positive ACS patients had involvement of multiple organ systems in addition to cardiac manifestations, thus indicating a systemic vascular damage. In comparison to recovered COVID-19 positive ACS patients, deceased COVID19 positive ACS patients had markedly elevated troponin values (factor increase in upper limit of the normal (ULN): 65.00 vs. $323.00 ; P=0.014$ ) and brain natriuretic peptide values (factor increase in ULN: 2.00 vs. $113.23 ; P=0.023$ ) accompanied by severely depressed left ventricular ejection fraction $(45.3 \pm 10.3 \%$ vs. $34.3 \pm 9.5 \% ; P=0.003)$ suggesting incremental SARS-CoV-2 related myocardial injury further aggravating ACS related heart failure.

The relatively low frequency of ACS in COVID-19 may in part explained by the fact that not all COVID-19 positive patients who exhibit ST-segment elevation undergo coronary angiography [3]. The concomitant occurrence of COVID19 and ACS might be responsible for the increased mortality. Pathophysiological mechanisms underlying COVID-19 related ACS events are unknown but might include acute plaque rupture or erosion facilitated by systemic inflammation, microvascular thrombosis due to hypercoagulability, and/or endothelial dysfunction [4]. The latter is known to play a key role in arterial hypertension and thrombosis and has recently been associated with COVID-19 [5]. In this respect, endotheliitis in COVID-19 might affect various vascular beds thereby increasing the susceptibility for thromboembolic and septic complications or multi-organ-failure [5]. Thus, myocardial ischemia due to ACS might be even aggravated by COVID-19 induced generalized microvascular dysfunction and systemic vascular damage leading to severe heart failure with unfavorable outcomes. Therefore, in addition to a guideline-directed ACS management, therapies to improve endothelial dysfunction might be considered in patients with COVID-19.

Funding None.

\section{Compliance with ethical standards}

Conflict of interest The authors report no conflicts of interest. 


\section{References}

1. Huang C, Wang Y, Li X, Ren L, Zhao J, Hu Y, Zhang L, Fan G, Xu J, Gu X, Cheng Z, Yu T, Xia J, Wei Y, Wu W, Xie X, Yin W, Li H, Liu M, Xiao Y, Gao H, Guo L, Xie J, Wang G, Jiang R, Gao Z, Jin Q, Wang J, Cao B (2020) Clinical features of patients infected with 2019 novel coronavirus in Wuhan. China Lancet 395(10223):497-506. https://doi.org/10.1016/S0140 -6736(20)30183-5

2. Shi S, Qin M, Shen B, Cai Y, Liu T, Yang F, Gong W, Liu X, Liang J, Zhao Q, Huang H, Yang B, Huang C (2020) Association of Cardiac injury with mortality in hospitalized patients with COVID-19 in Wuhan, China. JAMA Cardiol. https://doi. org/10.1001/jamacardio.2020.0950
3. Bangalore S, Sharma A, Slotwiner A, Yatskar L, Harari R, Shah B, Ibrahim H, Friedman GH, Thompson C, Alviar CL, Chadow HL, Fishman GI, Reynolds HR, Keller N, Hochman JS (2020) ST-segment elevation in patients with Covid-19-a case series. N Engl J Med. https://doi.org/10.1056/NEJMc2009020

4. Madjid M, Safavi-Naeini P, Solomon SD, Vardeny O (2020) Potential effects of coronaviruses on the cardiovascular system: a review. JAMA Cardiol. https://doi.org/10.1001/jamacardio .2020 .1286

5. Varga Z, Flammer AJ, Steiger P, Haberecker M, Andermatt R, Zinkernagel AS, Mehra MR, Schuepbach RA, Ruschitzka F, Moch H (2020) Endothelial cell infection and endotheliitis in COVID19. Lancet. https://doi.org/10.1016/S0140-6736(20)30937-5 